\title{
Myeloid Cells in Cancer Progression: Unique Subtypes and Their Roles in Tumor Growth, Vascularity, and Host Immune Suppression
}

\author{
Pampee P. Young • Shidrokh Ardestani • Bin Li
}

Received: 18 January 2010 / Accepted: 23 March 2010/Published online: 20 April 2010

(C) The Author(s) 2010. This article is published with open access at Springerlink.com

\begin{abstract}
Leukocytic infiltrates, particularly myeloid cells, can stimulate an anti-tumor immune response, but more often they stimulate tumor development, including promoting invasion, tumor growth, angiogenesis, and metastasis. Distinct myeloid phenotypes are being characterized that have been shown to promote tumor growth, angiogenesis, and metastasis. This review provides an overview of myeloid differentiation and spotlights specific pro-tumorogenic myeloid populations and their role in cancer progression. Efforts to characterize these pro-tumorogenic myeloid cell immunophenotypes may lead to novel targets for cancer therapy.
\end{abstract}

Keywords Myeloid derived suppressor cells · Tie-2 expressing monocytes · Tumor associated macrophages . Tumor microenvironment · Vascular leukocytes

$\begin{array}{ll}\text { Abbreviations } \\ \text { BM } & \text { Bone marrow } \\ \text { CMPs } & \text { Common myeloid progenitors }\end{array}$

P. P. Young $(\bowtie) \cdot$ S. Ardestani $\cdot$ B. Li

Departments of Pathology,

Vanderbilt University School of Medicine,

1161 21st Ave. South. C2217A MCN,

Nashville, TN 37232, USA

e-mail: pampee.young@vanderbilt.edu

P. P. Young

Internal Medicine, Vanderbilt University School of Medicine,

Nashville, TN, USA

P. P. Young

The Department of Veterans Affairs Medical Center,

Vanderbilt University School of Medicine,

Nashville, TN, USA

$\begin{array}{ll}\text { DCs } & \text { Dendritic cells } \\ \text { EPCs } & \text { Endothelial progenitor cells } \\ \text { GCV } & \text { Gancyclovir } \\ \text { GMPs } & \text { Granulocyte/macrophage progenitors } \\ \text { HSCs } & \text { Hematopoietic stem cells } \\ \text { MDPs } & \text { Myeloid dendritic-cell progenitors } \\ \text { MDSCs } & \text { Myeloid derived suppressor cells } \\ \text { MEPs } & \text { Megakaryocyte/erythroid Progenitors } \\ \text { MISCs } & \text { Myeloid immune suppressor cells } \\ \text { MPPs } & \text { Multi-potent progenitor cells } \\ \text { TAMs } & \text { Tumor associated macrophages } \\ \text { TEMs } & \text { Tie2 expressing monocytes } \\ \text { Tregs } & \text { Regulatory T cells }\end{array}$

\section{Introduction}

Tumor cells actively interact with each other and the surrounding interstitial tissue through cell-to-cell contacts as well as by both releasing and responding to soluble factors present in the tumor microenvironment. This interaction results in the generation of a population of non-malignant cells, collectively denoted as the "tumor stroma", that are uniquely primed to support growth and metastasis of the malignant population $[1,2]$. This "tumoreducated" stroma thus exhibits a unique phenotype that promotes the growth, invasion and metastasis of the malignant population $[3,4]$.

In recent years increasing attention has been focused on the role of the host inflammatory cells comprising the microenvironment stroma [5]. Specifically, myeloid cells are a major component of the inflammatory infiltrate frequently seen in primary tumors $[6,7]$. On one hand, myeloid/macrophage cells play a key role in the immune 
response by presenting antigens to lymphocytes to start the recognition and immune inductive process or by releasing regulatory molecules to stimulate lymphocyte functions [8]. As a component of the innate immune response, myeloid cells activate dendritic cells and natural killer (NK) cells that can initiate an anti-tumor response. Consistent with this role, in some mouse experimental models, syngeneic macrophages from tumor bearing mice inhibited melanoma growth in nude mice more than control macrophages [9]. However, often in both mouse models and in patients, the myeloid population can instead drive tumor progression by promoting neovascularization, metastasis, and immunosuppression $[4,6,7]$. Especially in human breast and cervical cancers, strong correlation has been observed between tumor macrophage density, as stained by a CD68 antibody, and aggressive phenotype [10-13]. However, in other types of human cancers, the data are often conflicting [14]. In some studies of human stomach, lung, and colorectal tumors, the degree of staining with a single macrophage marker, CD68, correlated with good prognosis $[14,15]$. The reason for the discrepancy correlating CD68 macrophage density and prognosis is not understood but may be related to the phenotype or nature of the different populations of myeloid cells present in the tumor, since CD68 stains mature macrophages but not all myeloid populations.

Myeloid cells circulate in peripheral blood as monocytes, which extravasate from the blood vessel to enter tissues [16]. The blood monocytes are young cells that already possess many of the functional capacities (migratory, chemotactic, and phagocytic) evident in more differentiated macrophages [17]. Extravasated monocytes can differentiate within tissues over time to become macrophages. It has been long appreciated that tissue macrophages display a range of functional and morphological phenotypes [18]. More recently, it can be argued that monocytes represent the circulating macrophage population and also exhibit unique properties and surface antigen expression in response to factors encountered while in circulation as well as after migration into specific tissues [19]. This review will address the role of myeloid cells in tumor growth and progression with emphasis on evaluation of myeloid cellderived endothelium (tumor vasculogenesis) and will evaluate a range of myeloid phenotypes implicated in these processes.

\section{Myeloid Differentiation}

Early in hematopoiesis multipotent, self renewing hematopoietic stem cells (HSCs) give rise to the multi-potent progenitor cells (MPPs) which can subsequently give rise to the common myeloid progenitors (CMPs). CMPs differen- tiate into macrophages and dendritic-cell progenitors (MDPs), granulocyte/macrophage progenitors (GMPs), megakaryocyte/erythroid Progenitors (MEPs), or basophile/ mast cells. MDPs give rise to monocytes and dendritic cells (DCs), however, GMPs have the ability to differentiate into monocytes and granulocytes [16, 20-23]. Figure 1 provides a schematic overview of this differentiation cascade and known species-specific antigen expression profile.

One of the important genes which is expressed during myeloid lineage differentiation is the PU.1 gene, an ETS transcription factor [20, 23-26]. PU.1 is critical during both B-lymphocyte and myeloid cell differentiation and its impaired expression results in both lymphocyte and monocyte/macrophage deficiency [23, 24]. Another important factor involved in monocyte to macrophage differentiation is the hematopoietic growth factor colony stimulating factor1 (CSF-1) (also known as CD115 and M-CSF). CSF-1 is the ligand for CSF-1R (c-fms, M-CSFR) which is expressed on monocytes, macrophages, and DCs. Mice deficient in CSF-1 or CSF-1R show dramatic reduction of blood monocytes $[27,28]$. Other important factors in differentiation of myeloid cells include transcription factors such as IRF8, cmyc, and c-myb [20, 27, 29].

Circulating immature myeloid cells, monocytes, are a heterogeneous population which can be divided into different subtypes based on their morphology and surface markers (Fig. 1). The two major subsets of monocytes include a short lived "inflammatory" and a long lived "resident" monocyte [19, 30-32]. Whereas some antigens such as CD11b [33] and CD163 [34, 35] have been characterized on both circulating monocytes and tissue macrophages, other phenotypic markers such as CD68 [36-38] (human) and F4/80 [39] (mouse) are restricted to tissue macrophages and are absent from the circulating myeloid (monocyte) populations [35]. The exact nature of what determines the differentiation of MDPs into different subsets such as inflammatory or resident monocytes is not clear and requires further investigation.

"Inflammatory" monocytes are defined by their expression of $\mathrm{Ly}_{6 \mathrm{c}^{+}} \mathrm{CX} 3 \mathrm{CR}^{\text {low }}, \mathrm{CCR}^{+}, \mathrm{CD} 2 \mathrm{~L}^{+}$(see Fig. 1), and $\mathrm{CD} 16^{-} \mathrm{CD} 14^{\text {high }}$ (only human) and are generated directly from MDPs in bone marrow (BM). They can recirculate between peripheral blood and BM [17, 19, 30-32]. Once they enter the peripheral circulation, inflammatory monocytes migrate into the inflamed tissues and presumably become "inflammatory" (i.e. M1) macrophages and DCs in the presence of inflammatory chemokines to further activate immune response [16, 17, 32, 40]. High level expression of CCR2 and $\mathrm{CD} 62 \mathrm{~L}$, which are receptors for the chemokine MCP-1/CCL2 by monocytes, facilitates the appropriate homing to injured tissue [19, 41-43].

The second major population of monocytes, "resident" monocytes, are characterized by a number of markers such 


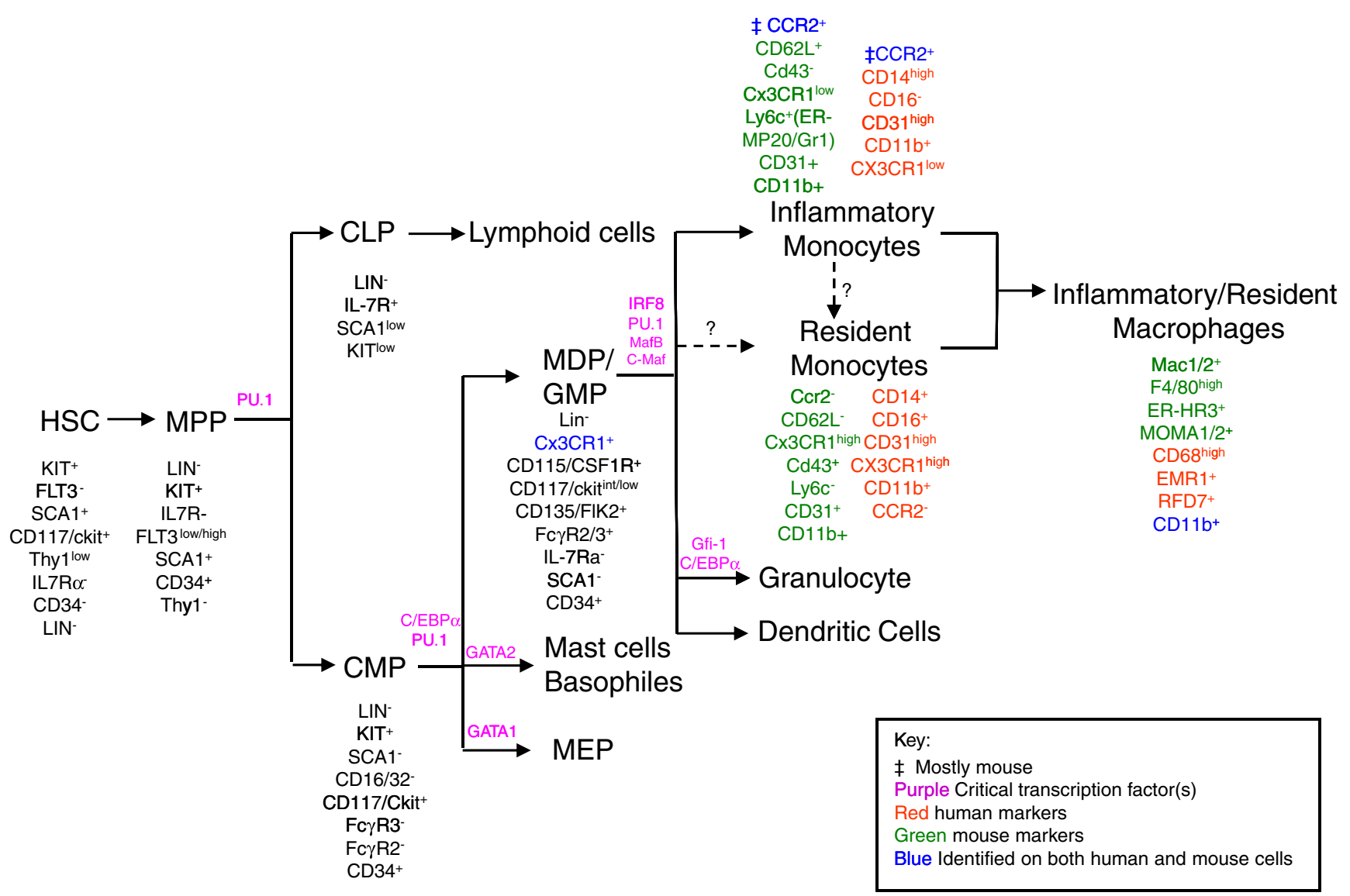

Fig. 1 Scheme of hematopoietic lineage differentiation. Depiction of the stages of myelopoiesis and associated surface antigen expression. Abbreviations: HSCs, Hematopoietic Stem Cells; MPPs, MultiPotential Progenitors; CLPs, Common Lymphoid Progenitors; GMPs, Granulocyte/

as $\mathrm{CX} 3 \mathrm{CR} 1^{\text {high }}$, $\mathrm{Ly}_{6 \mathrm{c}}^{-}, \mathrm{CCR} 2^{-}, \mathrm{CD}^{2} 2 \mathrm{~L}^{-}$(murine and human), $\mathrm{CD}^{+} 6^{+}, \mathrm{CD}^{+} 4^{+}$(mainly in human) $[16-18,31$, 32, 44, 45]. Resident monocytes home to non-inflamed tissues such as to liver, lung, brain, and spleen to comprise the resident macrophage population. They can also circulate in the peripheral blood and participate in the resolution of inflammation, wound healing, or in the removal of cell debris $[16,31,40,46,47]$. These populations of monocytes migrate into tissues as a result of high level expression of CX3CR1, which is the receptor of CX3CL1 ligand, a highly expressed transmembrane chemokine in endothelial cells $[19,48]$. It is not clear how the classification of monocytes and macrophages into resident and inflammatory subpopulations relate to the generation of the specific myeloid subpopulations described in this review, such as Tie2-expressing monocytes (TEMs) or myeloid derived suppressor cells (MDSCs). In the following sections, we will discuss recent characterizations of both immature and mature myeloid subpopulations linked to specific immunological and physiological function that lead to tumor promotion (Fig. 2).
Macrophage Progenitors; MEPs, Megakaryocyte/Erythroid Progenitors; CMPs, Common Myeloid Progenitors; MDPs, Macrophage and Dendritic-cell Progenitors. See [16, 18-25, 30, 32, 37, 39, 40, 44, 45, 112-120]

\section{Myeloid Phenotypes and Tumor Progression}

\section{Tumor Associated Macrophages (TAMs)}

The "TAMs" nomenclature broadly denotes the tumorassociated macrophage population. Studies to quantify TAM density in various malignancies have almost exclusively utilized the markers CD68 (human) or F4/80 (mouse). Many human studies have shown a positive correlation between tumor macrophage density and blood vessel density $[12,49,50]$. The mechanism(s) by which TAMs are believed to play a role in angiogenesis is primarily by secreting proangiogenic factors, such as VEGF-A, VEGF-C, and bFGF [51]. Expression of these genes by macrophages in tumors is regulated, at least in part, by hypoxia-mediated upregulation of transcription factors such as HIF $1 \alpha$ and HIF-2 $\alpha[49,52]$. In addition macrophages secrete many proteases including MMP-2, MMP-7, MMP-9, and MMP-12, that can support neoangiogenesis by facilitating matrix turnover [53, 54]. Matrix degradation enhances bioavailability of matrix-bound growth 


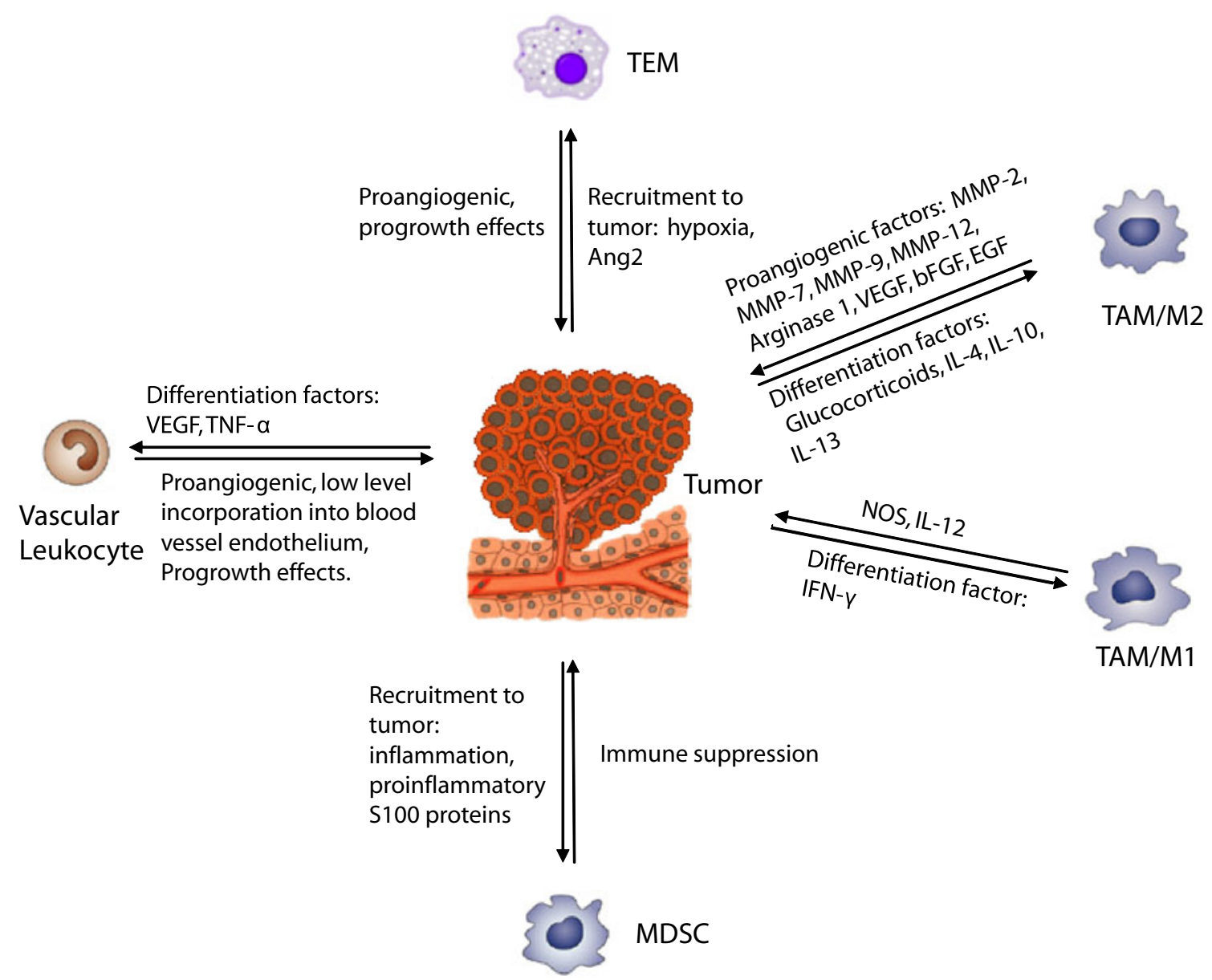

Fig. 2 Schematic of interactions between tumor and bone marrow derived myeloid subtypes. Noted are reported effects of the specific population on tumor growth. Factors that are involved in recruitment to tumor site are denoted for some populations, (i.e. TEMs and

factors and facilitates migration of vascular cells into new areas [55]. In addition to this proangiogenic function, it is hypothesized that TAMs serve as immunosuppressive cells to facilitate tumor evasion of the host immune system [56, 57]. Compared to thioglycolate-elicited peritoneal macrophages, TAMs secrete less interleukin (IL)-12 and other inflammatory cytokines and more immunosuppressive IL-10 [56]. As peritoneal, thioglycolate-stimulated macrophages were used for these comparative studies, it is not clear if the less inflammatory chemokine profile of TAMs is unique to tumors and distinct from other, non-malignant tissue- or wound- associated macrophages [56, 57].

Two major functional states have been described for tissue macrophages and named as M1 and M2 [58]. M1 macrophages represent classically activated macrophages that are induced by interferon- $\gamma$ alone or in combination with lipopolysaccharide and tumor necrosis factor- $\alpha$ [59]. M1 macrophages produce high levels of inducible nitric oxide synthase and pro-inflammatory cytokines, such as IL-12, and are generally considered as part of the inflamma-
MDSCs) that are present in the circulation of tumor-bearing animals. By contrast, vascular leukocytes and TAMs are primarily described within tumor tissues. Hence, factors involved in their generation in situ are noted

tory response, such as seen in infection [59-61]. In contrast, glucocortigoids, IL-4, IL-13 and IL-10 induce a distinct M2 macrophage phenotype that express arginase I and EGF, promote angiogenesis, favor tissue remodeling and invasion, and are able to inhibit the inflammatory response through the secretion of IL-10 [59, 60, 62]. Thus M2 macrophages would be hypothesized to exert a tumor-promoting activity by inhibiting the host immune response. Since a high density of $\mathrm{CD}^{+} 8^{+}$, intra-tumoral macrophages are associated with poor prognosis in a number of human tumors (i.e. cervix, and breast [63] [64], this association, in conjunction with the reported "anti-inflammatory" cytokine array profile of macrophages isolated from mouse tumors (i.e. IL-4, IL-13, etc.), are used to support the hypothesis that TAMs represent an M2-polarized macrophage [60]. Many studies have performed partial characterizations of TAMs, such as by showing the absence of macrophage NOS staining, to further support a predominant M2 phenotype [60]. However, aspects such as decreased expression of NOS, are also attributed to MDSCs $[65,66]$. Moreover, it is not clear if a 
decreased M1/M2 ratio reported among TAMs is distinct from other tissue resident macrophages. This is relevant since, unlike TAMs, tissue resident macrophages do not play an active role in promoting angiogenesis or secreting matrix proteases. As we gain greater understanding of tumor associated myeloid phenotypes, the M1 and M2 designations will likely be further refined.

\section{Myeloid Derived Suppressor Cells (MDSCs)}

MDSCs (also known as myeloid immune suppressor cells, MISCs) are a myeloid population which are significantly increased in the spleen and BM of tumor-bearing mice or in peripheral circulation of cancer patients and inhibit host immune function [65-67]. In mice, MDSCs express CD11b and Gr-1 [65]. CD11b is expressed by both circulating immature myeloid cells such as monocytes as well as macrophages (Fig. 1). Gr-1 is a murine specific antibody that recognizes both Ly-6G, expressed only on granulocytes, and Ly-6C, expressed on myeloid cells as well as some NK cells [65]. Evaluation of GR-1 ${ }^{+}$MDSCs using antibodies that distinguish between Ly-6C and Ly-6G have identified two MDSC subsets: Co-expression of CD11b and Ly- $6 \mathrm{G}^{+} / \mathrm{Ly}-6 \mathrm{C}^{\text {high }}$ identify primarily monocytic lineage cells whereas CD11b and $\mathrm{Ly}-6 \mathrm{G}^{+} / \mathrm{Ly}-6 \mathrm{C}^{\text {low }}$ identify granulocytic lineage cells $[65,68]$. Both subsets are increased in mice harboring tumors, although the larger increase was observed in the granulocytic subset [68]. In mouse tumor models, the levels of MDSCs in spleen and peripheral circulation appear to increase substantially in the presence of tumor to levels often greater than 50\% [65]. However, within tumors MDSCs constitute a modest portion of the leukocyte population within tumors $(<5 \%)$ [69].

In humans, "MDSC" populations have been reported on the basis of their ability to mediate immune suppression using a variety of different markers such as $\mathrm{CD} 11 \mathrm{~b}^{+} / \mathrm{CD} 14^{-}$, only as $\mathrm{CD}_{1} 4^{+}$, or $\mathrm{CD} 34^{+}$, although studies have also described a $\mathrm{CD} 14^{+} / \mathrm{HLA}-\mathrm{DR}^{-/ \text {low }}$ population [70-72]. The murine MDSC markers, $\mathrm{CD}_{11} \mathrm{~b}^{+} / \mathrm{Gr}-1^{+}$, are present on both immature (monocyte) and mature (macrophage) populations and have been studied in the context of both peripheral circulation as well as associated with tumor tissue. By contrast, human "MDSCs" are characterized by more immature markers and, hence, have been enumerated primarily in peripheral blood of cancer patients [70-72]. The diversity of circulating MDSC phenotype in humans suggests that a assorted population of myeloid cells may exhibit immune inhibitory functions and that the immuneinhibitory phenotype is likely to be driven by interplay between host and tumor-derived signals [65, 67].

The $\mathrm{CD} 11 \mathrm{~b}^{+} / \mathrm{Gr}-1^{+}$murine MDSCs were initially defined by their ability to suppress the immune system through multiple immune effectors, including direct mod- ulation of $\mathrm{T}$ cells and NK cell activation and also by inducing regulatory $\mathrm{T}$ cells (Tregs) through a number of mechanisms [73, 74]. Important factors that contribute to the suppressive activity of MDSCs are production of high levels of reactive oxygen species and peroxynitrite, both of which suppress T cell function [75]. Interestingly, blocking MDSC-derived reactive oxygen species led to their differentiation into macrophages and dendritic cells, suggesting that this may be a mechanism by which to target MDSCs [75]. Another potential method of MDSC-mediated T cell suppression is through the high intracellular expression of arginase, which causes a reduction in arginine used in $\mathrm{T}$ cell activation [72, 76, 77]. MDSCs also activate NOS and together with arginase, can generate superoxides that also facilitate T cell apoptosis [78]. Such effects on T cells serve to suppress anti-tumor immunity and also modulate the behavior of antigen presenting cells (DCs and macrophages) [79]. Moreover, treatment of tumor bearing mice with anti GR-1 antibody (which recognize both MDSCs and neutrophils) resulted in delayed tumor growth, providing tantalizing data to further explore the effect of targeting MDSCs for tumor therapy [80].

MDSCs may also play a broader role in modulating tumorrelated inflammation. MDSCs modulate cytokine production of macrophages, skewing them towards decreased production of IL-12, potentially through Toll-like receptor 4 signaling [81]. Gr- $1^{+}$myeloid populations were also found to promote tumor angiogenesis by expressing MMP-9 and VEGF and play a role in the angiogenic switch leading to the development of islet carcinoma [82]. More recently, direct incorporation of $\mathrm{CD} 1 \mathrm{~b}^{+} / \mathrm{Gr}-1^{+}$cells into vascular endothelium were reported when such cells were admixed with tumor cells prior to implantation, albeit the frequency of endothelial differentiation of MDSCs was not reported [69].

Currently, the majority of studies addressing the immunosuppressive function of MDSCs utilize splenic or peripheral blood-derived cells obtained from tumor-bearing mice [65]. It is not clear if MDSCs within tumors function similarly as immune-suppressive cells. More studies are needed to confirm and further understand changes in surface phenotype and/or function of murine MDSCs once they are recruited to the tumor. It will also be important to clarify and further characterize the human MDSCs and define if the aspects attributed to murine $\mathrm{CD} 11 \mathrm{~b}^{+} / \mathrm{Gr}-1^{+}$MDSCs are relevant to human cancers.

Vascular Leukocytes/Myeloid-Endothelial Biphenotypic Cells

\section{Myeloid Plasticity}

Several groups, including our own, have shown that circulating human or mouse monocytes can be induced in 
culture to express endothelial markers [e.g. VE-cadherin (CD156), flk-1(VEGFR2/KDR), von Willebrand Factor (vWF), tie2, and endothelial lectins] and an endothelial phenotype (network formation, synthesis of eNOS, and Weibel Palade bodies) [83-86]. Although this population shows limited proliferative potential, these cells can home to sites of ischemia and tumor where they demonstrate significant proangiogenic effects [87]. Importantly, they are also capable, at modest levels, of incorporating into functional neovasculature, i.e., vasculogenesis [87-89]. In fact, many studies attempting to generate and/or measure endothelial progenitor cells (EPCs) have utilized short term adhesion of mononuclear cells to fibronectin-coated dishes which primarily selects for endothelial differentiation of circulating monocytes [90]. In vitro culture of monocytes in endothelial promoting media initially generated cells that co-expressed both endothelial and myeloid markers [85]. Upon prolonged culture ( $>30 \mathrm{~d})$, hematopoietic and myeloid marker expression were eventually downregulated but endothelial antigen expression was retained in culture [85].

Additionally, myeloid to endothelial plasticity has also been demonstrated in vivo in several preclinical models. Specifically, adoptive transfer of highly enriched immature myeloid progenitors resulted in these myeloid cell-derived blood vessels in the recipient liver [91]. Monocyte-derived endothelial-like cells were shown to engraft in tumor vessels in low numbers. Vascular plasticity of myeloid cells has been also described during ischemic injury in murine hearts overexpressing the chemokine MCP-1/CCL2 [92], as well as in other contexts. Goodell and colleagues, for example, showed that myofibers generated in an inflammatory nidus can be derived at low levels from hematopoietic transdifferentiation and that this occurs through a myeloid intermediate [93]. Two other independent studies show that myeloid progenitors transdifferentiate into lymphatic endothelial cells in inflammatory/repair tissues [94, 95].

To better define if endothelial differentiation from myeloid progenitors occurred more efficiently from mature versus immature myeloid progenitors, our group used various myeloid populations isolated from transgenic mice that expressed lacZ under the control of an endothelial cell promoter [85]. These data supported that myeloid to endothelial differentiation occurred with $\sim 4$-fold greater efficiency from early myeloid progenitors, not differentiated progeny such as macrophages [85]. Adoptive transfer studies using a different model system also further supported the notion that more immature myeloid progenitors made a greater contribution to host hepatic vasculature [91].

\section{Vascular Leukocytes in Tumors}

Although a growing body of data (as discussed above) showed that cultured monocytes can be induced to co- express myeloid and endothelial antigens, evidence for the existence of a myeloid/endothelial biphenotypic population (also called vascular leukocytes) in vivo was introduced more recently during evaluation of both mouse and human ovarian cancer tissue. A tumor associated $\mathrm{CD} 11 \mathrm{c}^{+}$myeloid population was identified that co-expressed endothelial markers, $\mathrm{P} 1 \mathrm{H} 12$ and VE-cadherin, in murine ovarian cancers $[69,96]$. Myeloid/endothelial biphenotypic populations were also identified in murine sarcoma and melanomas that comprised around $2-5 \%$ of tumor-associated leukocytes [85]. In this study vascular leukocytes were not detected in normal mouse or human peripheral blood or bone marrow. This suggests that the biphenotypic cell may not be part of the normal differentiation cascade of the hematopoietic progenitor but may be generated from vascular leukocytes (e.g. myeloid cells) present at the tumor site by specific local cues [85]. In analysis of 10 consecutive advanced stage human ovarian cancers, similar populations of biphenotypic cells co-expressing CD14 monocyte marker and multiple endothelial markers were also identified to comprise $52 \%$ of tumor-associated leukocytes $\left(\mathrm{CD} 45^{+}\right.$ cells) $[69,96]$.

Isolated vascular leukocytes from mouse and human tumor tissue were capable of generating functional vessels when reimplanted in vivo within matrigel plugs [97]. However, it is unclear the extent of contribution of vascular leukocytes to functional, in situ tumor vasculature [97, 98]. Co-localization of myeloid markers with functional vessels as well as increased frequency of bone marrow derived vessels under conditions that were shown to increase vascular leukocytes (such as increased VEGF or TNF- $\alpha$ ) have been reported in tumors, however, the relative direct contribution of vascular leukocytes to tumor vasculature is unknown but suspected to be very low [97, 98]. In one study, the level of myeloid cell-derived tumor vasculature was found to be dependent of the type of tumor, present in lewis lung carcinoma-derived tumors but absent in mouse melanoma, despite the fact that relatively similar levels of microvasculature was evident [99]. Moreover, it was found that site specific expression of stromal derived factor- $1 \alpha$ (SDF-1 $\alpha /$ CXCL12a) was necessary for neovasculogenesis from myeloid cells in tumors [99]. Despite low direct contribution to vasculature, vascular leukocytes represent a myeloid population that promotes tumor growth. Admixing myeloid cells with tumors under conditions that promote generation of vascular leukocytes (ie VEGF or TNF- $\alpha$ expressing tumors) resulted in larger, highly vascularized tumors $[97,98]$. In addition to their significant contribution to tumor angiogenesis as well as some impact on tumor vasculogenesis, it will be important to investigate if this population, like MDSCs, also exhibit immunosuppressive attributes as well as elucidate the mechanism by which vascular leukocytes promote tumor growth and vascularity. 


\section{Tie2 Expressing Monocytes (TEMs)}

A small (1-4\%) population of $\mathrm{CD} 11 \mathrm{~b}^{+}$cells expressing Tie2, an endothelial enriched gene encoding angiopoietin receptor tyrosine kinase, were identified in mouse mammary tumors [100, 101]. These were named Tie2 expressing monocytes (TEMs), in part because they were initially characterized as expressing $\mathrm{CD}_{11} \mathrm{~b}^{+}$, a marker present on circulating monocytes (though $\mathrm{CD} 11 \mathrm{~b}$ can also be present on differentiated macrophages, see Fig. 1) [65, 101, 102]. The monocyte nomenclature suggests that these cells represent an immature myeloid lineage, which is supported by the fact that $\mathrm{CD} 11 \mathrm{~b}^{+} / \mathrm{Tie}^{+}$TEMs were also identified in the peripheral circulation of both human and mice [103]. Enriched populations of peripheral blood-derived TEMs exhibited proangiogenic properties in a matrigel plug assay, albeit these cells were not compared directly with a myeloidenriched population depleted of TEMS but instead to peripheral blood mononuclear cells (which would contain large numbers of lymphocytes, some granulocytes as well as myeloid cells) depleted of TEMs [101]. In a more recent study, TEMs isolated directly from mouse mammary tumors were characterized to express similar levels of F4/80 antigen as TAMs, suggesting that TEMs isolated from the tumor tissue itself may represent a well-differentiated myeloid (macrophage) population [104]. Moreover, it is not known if circulating TEMs exhibit a distinct functional phenotype from tumor-associated TEMs [104].

TEMs were uniquely enriched in the tumor periphery in association with blood vessels in mouse tumors [69, 96, 101]. Despite their low abundance in comparison to the total tumor myeloid population, TEMs displayed a striking proangiogenic phenotype when compared to $\mathrm{CD}_{1} 1 \mathrm{~b}^{+} / \mathrm{Tie}^{-}$ population [100]. Selective ablation of TEMS in mouse LLC, glioma, mammary [101, 105] with a suicide gene induced by the drug gancyclovir (GCV) at the time of tumor implantation resulted in $\sim 2$-fold delayed growth and significantly reduced angiogenesis [105]. Importantly, despite expression of an endothelial marker, Tie2, murine tumor-derived TEMs were shown by extensive genomic characterization to differ markedly from endothelial cells and from $\mathrm{CD} 11 \mathrm{~b}^{+} / \mathrm{Gr}-1^{+}$(MDSCs) derived from spleen, but to be similar to TAMs [104]. The differences identified between MDSCs and TEMs may reflect that splenic, instead of tumor-associated, MDSCs were used for the comparative analysis [104]. It is likely, that myeloid populations isolated from peripheral blood or spleen represent a more immature cell type than ones isolated from tumor tissue suspensions. However, the lack of antigenic and genomic overlap between endothelial cells and TEMs combined with the clear similarities between TEMs and other myeloid and hematopoietic cells suggest that TEMs, unlike vascular leukocytes, are unlikely to undergo trans- differentiation into endothelial cells $[69,96]$. In fact, although TEMs were found closely associated with tumor vasculature, evidence of direct contribution to vessel endothelium was absent $[69,96]$. These data are further supported by our study which identified TNF- $\alpha$ as an important tumor-derived factor that enhance tumorassociated vascular leukocytes by promoting in situ differentiation of endothelial differentiation of myeloid cells [98]. Importantly, TNF- $\alpha$ did not increase tumor associated $\mathrm{CD} 11 \mathrm{~b}^{+} / \mathrm{Tie}^{+}$or $\mathrm{F} 4 / 80^{+} / \mathrm{Tie}^{+}$TEMs.

\section{Myeloid Educating and Recruiting Signals}

Circulating blood monocytes are recruited to the tumor by a range of growth factors and chemokines, often produced by the tumors themselves [106]. Other inflammatory cells, such as B lymphocytes, have been implicated in producing trophic factors that promote macrophage recruitment [107]. Following recruitment, it is widely accepted that the tumor/ myeloid cell crosstalk (Fig. 2) educate recruited myeloid cells towards a tumor-promoting phenotype(s), although the nature of these phenotypes is not completely understood $[5,108]$. Understanding the mechanisms by which protumorogenic, myeloid immunophenotypes are generated either in circulation or within the tumor milieu can be used to develop novel anti-tumor therapy [108]. Hence, this area of study is on the forefront of cancer research [108]. While a number of factors have been identified that recruit myeloid cells to tumor sites (i.e., VEGF, PIGF, MCP-1/ CCL2, SDF- $1 \alpha /$ CXCL12a, and $\beta$-defensins) [109], there is much less known about tumor-derived signals that modulate the generation of unique myeloid subtypes during cancer growth. Tumor-derived VEGF and TNF- $\alpha$ have been shown to increase in situ differentiation of tumorassociated vascular leukocytes [97, 98]. It is suggested that the complement of cytokines (high IL-4, 10 and 13) may skew the TAM population to an M2 phenotype [110]. A recent study has shown that IL-4 and IL-13, especially derived from $\mathrm{Th} 2$-polarized $\mathrm{CD}^{+} \mathrm{T}$ lymphocytes, promote some aspects of the M2-type immunosuppressive phenotype in a context-dependent manner [62]. Other aspects of the M2 immunosuppressive phenotype may be regulated by NF-kB signaling whereas the proangiogenic properties of the M2 TAMs are regulated by other signals [111]. Other unique myeloid populations, such as TEMs and MDSCs, are thought to be present in the circulation and get recruited to the tumor site $[66,101,112]$. Many factors, such as VEGF, have been implicated with accumulation of several myeloid subtypes [4, 97]. Increased TEM density was found in association with tumor hypoxia and Ang2 secretion [100]. Many proinflammatory factors (i.e. VEGF, GM-CSF, IL-1b, IL-6, and S-100 proteins) were associated with increased circulating MDSCs [66]. More studies are 
needed to understand how tumor/host crosstalk generates both systemic and local signals that enhance specific myeloid subtypes.

\section{Conclusion}

TAMs, MDSCs, TEMs and vascular leukocytes are myeloid populations implicated in promoting tumor progression (Fig. 2). Although each of these myeloid subpopulations demonstrates some characteristic phenotype/ antigen profile, these subpopulations also display significant diversity in function and characterization among different model systems and likely represent a heterogeneous population. Human MDSCs, as an example, have been characterized with different sets of surface markers [70-72]. In different tumor models MDSCs mediate immune suppression by distinct mechanisms [65]. Moreover, there is a great deal of overlap in the pro-angiogenic and/or immunosuppressive functions of most of these myeloid subtypes. For example, in addition to vascular leukocytes, one study suggests that MDSCs may also be capable of contributing to blood vessels by endothelial differentiation [69]. The role of myeloid cells as an "endothelial progenitor cell (EPC)" remains controversial; both additional data and elucidation of the impact of myeloid-derived endothelium to tumor growth and progression are needed. Future studies to better characterize and define the functional roles of these protumorogenic myeloid subsets will hopefully elucidate the complex biology of myeloid cells during tumor progression.

Acknowledgements This work was supported by grants BX000209 and HL088424 (to PPY) and training grant 5T32HL069765-08 (to SA).

Open Access This article is distributed under the terms of the Creative Commons Attribution Noncommercial License which permits any noncommercial use, distribution, and reproduction in any medium, provided the original author(s) and source are credited.

\section{References}

1. Whiteside TL (2008) The tumor microenvironment and its role in promoting tumor growth. Oncogene 27:5904-5912

2. Goldoni S, Iozzo RV (2008) Tumor microenvironment: modulation by decorin and related molecules harboring leucine-rich tandem motifs. Int J Cancer 123:2473-2479

3. Joyce JA, Pollard JW (2009) Microenvironmental regulation of metastasis. Nat Rev Cancer 9:239-252

4. Pollard JW (2008) Macrophages define the invasive microenvironment in breast cancer. J Leukoc Biol 84:623-630

5. Balkwill F, Charles KA, Mantovani A (2005) Smoldering and polarized inflammation in the initiation and promotion of malignant disease. Cancer Cell 7:211-217

6. Balkwill F, Coussens LM (2004) Cancer: an inflammatory link. Nature 431:405-406
7. Murdoch C, Muthana M, Coffelt SB et al (2008) The role of myeloid cells in the promotion of tumour angiogenesis. Nat Rev Cancer 8:618-631

8. Mosser DM, Edwards JP (2008) Exploring the full spectrum of macrophage activation. Nat Rev Immunol 8:958-969

9. Adelman DC, Erickson KL, Gershwin E (1983) Macrophagemediated inhibition of melanoma cell growth in nude mice. Pathobiology 51:165-171

10. Leek RD, Lewis CE, Whitehouse R et al (1996) Association of macrophage infiltration with angiogenesis and prognosis in invasive breast carcinoma. Cancer Res 56:4625-4629

11. Volodko N, Reiner A, Rudas M et al (1998) Tumour-associated macrophages in breast cancer and their prognostic correlations. Breast 7:99-105

12. Salvesen HB, Akslen LA (1999) Significance of tumourassociated macrophages, vascular endothelial growth factor and thrombospondin-1 expression for tumour angiogenesis and prognosis in endometrial carcinomas. Int J Cancer 84:539-543

13. Fujimoto J, Sakaguchi H, Aoki I et al (2000) Clinical implications of expression of interleukin 8 related to angiogenesis in uterine cervical cancers. Cancer Res 60:2632-2635

14. Bingle L, Brown NJ, Lewis CE (2002) The role of tumourassociated macrophages in tumour progression: implications for new anticancer therapies. J Pathol 196:254-265

15. Nakayama Y, Nagashima N, Minagawa N et al (2002) Relationships between tumor-associated macrophages and clinicopathological factors in patients with colorectal cancer. Anticancer Res 22:4291-4296

16. Auffray C, Sieweke MH, Geissmann F (2009) Blood monocytes: development, heterogeneity, and relationship with dendritic cells. Annu Rev Immunol 27:669-692

17. Varol C, Yona S, Jung S (2009) Origins and tissue-contextdependent fates of blood monocytes. Immunol Cell Biol 87:30 38

18. Gordon S, Taylor PR (2005) Monocyte and macrophage heterogeneity. Nat Rev Immunol 5:953-964

19. Geissmann F, Jung S, Littman DR (2003) Blood monocytes consist of two principal subsets with distinct migratory properties. Immunity 19:71-82

20. Rosenbauer F, Tenen DG (2007) Transcription factors in myeloid development: balancing differentiation with transformation. Nat Rev Immunol 7:105-117

21. Orkin SH (2000) Diversification of hematopoietic stem cells to specific lineages. Nat Rev Genet 1:57-64

22. Kondo M, Wagers AJ, Manz MG et al (2003) Biology of hematopoietic stem cells and progenitors: implications for clinical application. Annu Rev Immunol 21:759-806

23. Dakic A, Metcalf D, Di Rago L et al (2005) PU.1 regulates the commitment of adult hematopoietic progenitors and restricts granulopoiesis. J Exp Med 201:1487-1502

24. Iwasaki H, Somoza C, Shigematsu H et al (2005) Distinctive and indispensable roles of PU.1 in maintenance of hematopoietic stem cells and their differentiation. Blood 106:1590-1600

25. McKercher SR, Torbett BE, Anderson KL et al (1996) Targeted disruption of the PU.1 gene results in multiple hematopoietic abnormalities. EMBO J 15:5647-5658

26. Nerlov C, Graf T (1998) PU.1 induces myeloid lineage commitment in multipotent hematopoietic progenitors. Genes Dev 12:2403-2412

27. Dai XM, Ryan GR, Hapel AJ et al (2002) Targeted disruption of the mouse colony-stimulating factor 1 receptor gene results in osteopetrosis, mononuclear phagocyte deficiency, increased primitive progenitor cell frequencies, and reproductive defects. Blood 99:111-120

28. Cecchini MG, Dominguez MG, Mocci S et al (1994) Role of colony stimulating factor-1 in the establishment and regulation of 
tissue macrophages during postnatal development of the mouse. Development 120:1357-1372

29. Sullivan J, Feeley B, Guerra J et al (1997) Identification of the major positive regulators of c-myb expression in hematopoietic cells of different lineages. J Biol Chem 272:1943-1949

30. Grage-Griebenow E, Flad HD, Ernst M (2001) Heterogeneity of human peripheral blood monocyte subsets. J Leukoc Biol 69:11-20

31. Sunderkotter C, Nikolic T, Dillon MJ et al (2004) Subpopulations of mouse blood monocytes differ in maturation stage and inflammatory response. J Immunol 172:4410-4417

32. Swirski FK, Nahrendorf M, Etzrodt M et al (2009) Identification of splenic reservoir monocytes and their deployment to inflammatory sites. Science 325:612-616

33. Kawai K, Tsuno NH, Matsuhashi M et al (2005) CD11bmediated migratory property of peripheral blood B cells. J Allergy Clin Immunol 116:192-197

34. Hogger P, Sorg C (2001) Soluble CD163 inhibits phorbol ester-induced lymphocyte proliferation. Biochem Biophys Res Commun 288:841-843

35. Mikami M, Sadahira Y, Suetsugu Y et al (2004) Monocyte/ Macrophage-specific marker CD163+ histiocytic sarcoma: case report with clinical, morphologic, immunohistochemical, and molecular genetic studies. Int J Hematol 80:365-369

36. Elner SG, Elner VM, Nielsen JC et al (1992) CD68 antigen expression by human retinal pigment epithelial cells. Exp Eye Res 55:21-28

37. Holness CL, Simmons DL (1993) Molecular cloning of CD68, a human macrophage marker related to lysosomal glycoproteins. Blood 81:1607-1613

38. Pulford KA, Sipos A, Cordell JL et al (1990) Distribution of the CD68 macrophage/myeloid associated antigen. Int Immunol 2:973-980

39. Khazen W, M'Bika JP, Tomkiewicz C et al (2005) Expression of macrophage-selective markers in human and rodent adipocytes. FEBS Lett 579:5631-5634

40. Nahrendorf M, Swirski FK, Aikawa E et al (2007) The healing myocardium sequentially mobilizes two monocyte subsets with divergent and complementary functions. J Exp Med 204:3037-3047

41. Boring L, Gosling J, Chensue SW et al (1997) Impaired monocyte migration and reduced type 1 (Th1) cytokine responses in C-C chemokine receptor 2 knockout mice. J Clin Invest 100:2552-2561

42. Serbina NV, Pamer EG (2006) Monocyte emigration from bone marrow during bacterial infection requires signals mediated by chemokine receptor CCR2. Nat Immunol 7:311-317

43. Tsou CL, Peters W, Si Y et al (2007) Critical roles for CCR2 and MCP-3 in monocyte mobilization from bone marrow and recruitment to inflammatory sites. J Clin Invest 117:902-909

44. Varol C, Landsman L, Fogg DK et al (2007) Monocytes give rise to mucosal, but not splenic, conventional dendritic cells. J Exp Med 204:171-180

45. Yona S, Jung S (2010) Monocytes: subsets, origins, fates and functions. Curr Opin Hematol 17(1):53-59

46. Arnold L, Henry A, Poron F et al (2007) Inflammatory monocytes recruited after skeletal muscle injury switch into antiinflammatory macrophages to support myogenesis. J Exp Med 204:1057-1069

47. Auffray C, Fogg D, Garfa M et al (2007) Monitoring of blood vessels and tissues by a population of monocytes with patrolling behavior. Science 317:666-670

48. Fong AM, Robinson LA, Steeber DA et al (1998) Fractalkine and CX3CR1 mediate a novel mechanism of leukocyte capture, firm adhesion, and activation under physiologic flow. J Exp Med 188:1413-1419

49. Leek RD, Talks KL, Pezzella F et al (2002) Relation of hypoxiainducible factor-2 alpha (HIF-2 alpha) expression in tumorinfiltrative macrophages to tumor angiogenesis and the oxidative thymidine phosphorylase pathway in Human breast cancer. Cancer Res 62:1326-1329

50. Lissbrant IF, Stattin P, Wikstrom P et al (2000) Tumor associated macrophages in human prostate cancer: relation to clinicopathological variables and survival. Int J Oncol 17:445-451

51. Lin EY, Pollard JW (2007) Tumor-associated macrophages press the angiogenic switch in breast cancer. Cancer Res 67:5064 5066

52. Chai CY, Chen WT, Hung WC et al (2008) Hypoxia-inducible factor-1alpha expression correlates with focal macrophage infiltration, angiogenesis and unfavourable prognosis in urothelial carcinoma. J Clin Pathol 61:658-664

53. Lewis CE, Pollard JW (2006) Distinct role of macrophages in different tumor microenvironments. Cancer Res 66:605-612

54. Hagemann T, Robinson SC, Schulz M et al (2004) Enhanced invasiveness of breast cancer cell lines upon co-cultivation with macrophages is due to TNF-alpha dependent up-regulation of matrix metalloproteases. Carcinogenesis 25:1543-1549

55. Giraudo E, Inoue M, Hanahan D (2004) An amino-bisphosphonate targets MMP-9-expressing macrophages and angiogenesis to impair cervical carcinogenesis. J Clin Invest 114:623-633

56. Biswas SK, Gangi L, Paul S et al (2006) A distinct and unique transcriptional program expressed by tumor-associated macrophages (defective NF-kappaB and enhanced IRF-3/STAT1 activation). Blood 107:2112-2122

57. Gottfried E, Faust S, Fritsche J et al (2003) Identification of genes expressed in tumor-associated macrophages. Immunobiology 207:351-359

58. Martinez FO, Helming L, Gordon S (2009) Alternative activation of macrophages: an immunologic functional perspective. Annu Rev Immunol 27:451-483

59. Mantovani A, Sica A, Sozzani S et al (2004) The chemokine system in diverse forms of macrophage activation and polarization. Trends Immunol 25:677-686

60. Sica A, Schioppa T, Mantovani A et al (2006) Tumourassociated macrophages are a distinct M2 polarised population promoting tumour progression: potential targets of anti-cancer therapy. Eur J Cancer 42:717-727

61. Ricardo SD, van Goor H, Eddy AA (2008) Macrophage diversity in renal injury and repair. J Clin Invest 118:3522-3530

62. DeNardo DG, Barreto JB, Andreu P et al (2009) CD4(+) T cells regulate pulmonary metastasis of mammary carcinomas by enhancing protumor properties of macrophages. Cancer Cell $16: 91-102$

63. O'Brien J, Schedin P (2009) Macrophages in breast cancer: do involution macrophages account for the poor prognosis of pregnancy-associated breast cancer? J Mammary Gland Biol Neoplasia 14:145-157

64. Hammes LS, Tekmal RR, Naud P et al (2007) Macrophages, inflammation and risk of cervical intraepithelial neoplasia (CIN) progression-clinicopathological correlation. Gynecol Oncol 105:157-165

65. Ostrand-Rosenberg S, Sinha P (2009) Myeloid-derived suppressor cells: linking inflammation and cancer. J Immunol 182:4499-4506

66. Serafini P, Borrello I, Bronte V (2006) Myeloid suppressor cells in cancer: recruitment, phenotype, properties, and mechanisms of immune suppression. Semin Cancer Biol 16:53-65

67. Bronte V, Serafini P, Apolloni E et al (2001) Tumor-induced immune dysfunctions caused by myeloid suppressor cells. J Immunother 24:431-446

68. Youn JI, Nagaraj S, Collazo M et al (2008) Subsets of myeloidderived suppressor cells in tumor-bearing mice. J Immunol 181:5791-5802

69. Yang L, DeBusk LM, Fukuda K et al (2004) Expansion of myeloid immune suppressor $\mathrm{Gr}+\mathrm{CD} 11 \mathrm{~b}+$ cells in tumor-bearing host directly promotes tumor angiogenesis. Cancer Cell 6:409-421 
70. Filipazzi P, Vanlenti HV et al (2007) Identification of a new subset of myeloid suppressor cells in peripheral blood of melanoma patients with modulation by a granulocyte-macrophage colony-stimulation factor-based antitumor vaccine. J Clin Oncology 25:2546-2553

71. Lathers DM, Achille N, Kolesiak K et al (2001) Increased levels of immune inhibitory CD34+ progenitor cells in the peripheral blood of patients with node positive head and neck squamous cell carcinomas and the ability of these CD34+ cells to differentiate into immune stimulatory dendritic cells. Otolaryngol Head Neck Surg 125:205-212

72. Zea AH, Rodriguez PC, Atkins MB et al (2005) Arginaseproducing myeloid suppressor cells in renal cell carcinoma patients: a mechanism of tumor evasion. Cancer Res 65:3044-3048

73. Serafini P, Mgebroff S, Noonan K et al (2008) Myeloidderived suppressor cells promote cross-tolerance in B-cell lymphoma by expanding regulatory $\mathrm{T}$ cells. Cancer Res 68:5439-5449

74. Gabrilovich DI, Nagaraj S (2009) Myeloid-derived suppressor cells as regulators of the immune system. Nat Rev Immunol 9:162-174

75. Corzo CA, Cotter MJ, Cheng P et al (2009) Mechanism regulating reactive oxygen species in tumor-induced myeloidderived suppressor cells. J Immunol 182:5693-5701

76. Nagaraj S, Gabrilovich DI (2008) Tumor escape mechanism governed by myeloid-derived suppressor cells. Cancer Res 68:2561-2563

77. Kusmartsev SA, Li Y, Chen SH (2000) Gr-1+ myeloid cells derived from tumor-bearing mice inhibit primary $\mathrm{T}$ cell activation induced through $\mathrm{CD} 3 / \mathrm{CD} 28$ costimulation. J Immunol 165:779-785

78. Mazzoni A, Bronte V, Visintin A et al (2002) Myeloid suppressor lines inhibit $\mathrm{T}$ cell responses by an NO-dependent mechanism. J Immunol 168:689-695

79. Sinha P, Clements VK, Bunt SK et al (2007) Cross-talk between myeloid-derived suppressor cells and macrophages subverts tumor immunity toward a type 2 response. J Immunol 179:977-983

80. Pekarek LA, Starr BA, Toledano AY et al (1995) Inhibition of tumor growth by elimination of granulocytes. J Exp Med 181:435-440

81. Bunt SK, Clements VK, Hanson EM et al (2009) Inflammation enhances myeloid-derived suppressor cell cross-talk by signaling through Toll-like receptor 4. J Leukoc Biol 85:996-1004

82. Nozawa H, Chiu C, Hanahan D (2006) Infiltrating neutrophils mediate the initial angiogenic switch in a mouse model of multistage carcinogenesis. Proc Natl Acad Sci U S A 103:12493-12498

83. Rehman J, Jingling L, Orschell CM et al (2003) Peripheral blood "endothelial progenitor cells" are derived from monocyte/ macrophages and secrete angiogenic growth factors. Circ 107:1164-1169

84. Romagnani P, Annunziato F, Liotta F et al (2005) CD14+CD34low cells with stem cell phenotype and functional features are the major source of circulating endothelial progenitors. Circ Res 97:314-322

85. Sharpe EE, Teleron AA, Li B et al (2006) The origin and in vivo significance of murine and human culture expanded endothelial progenitor cells (CE-EPCs). Am J Pathol 168:1710-1720

86. Ingram DA, Mead LE, Tanaka H et al (2004) Identification of a novel hierarchy of endothelial progenitor cells using human peripheral and umbilical cord blood. Blood 104:2752-2760

87. Raffi S, Lyden D (2003) Therapeutic stem and progenitor cell transplantation for organ vascularization and regeneration. Nature Med 9:702-712

88. Kalka C, Masuda H, Takahashi T et al (2000) Vascular endothelial growth factor 165 gene transfer augments circulating endothelial progenitor cells in human subjects. Circ Res 86:1198-1202
89. Yamaguchi J, Kusano KF, Masuo O et al (2003) Stromal cellderived factor-1 effects on ex vivo expanded endothelial progenitor cell recruitment for ischemic neovascularization. Circ 107:1322-1328

90. Yoder MC, Mead LE, Prater D et al (2007) Redefining endothelial progenitor cells via clonal analysis and hematopoietic stem/progenitor cell principals. Blood 109:1801-1809

91. Bailey AS, Willenbring H, Jiang S et al (2006) Myeloid lineage progenitors give rise to vascular endothelium. Proc Natl Acad Sci USA 103:13156-13161

92. Moldovan NI, Goldschmidt-Clermont PJ, Parker-Thornburg J et al (2000) Contribution of monocytes/macrophages to compensatory neovascularization: the drilling of metalloelastase-positive tunnels in ischemic myocardium. Circ Res 87:341-343

93. Camargo FD, Green R, Capetenaki Y et al (2003) Single hematopoietic stem cells generate skeletal muscle through myeloid intermediates. Nature Med 9:1520-1527

94. Kerjaschki D, Hurttary N, Raab I et al (2006) Lymphatic endothelial progenitor cells contribute to de novo lymphangiogenesis in human renal transplants. Nat Med 12:230-234

95. Maruyama K, Ii M, Cursiefen C et al (2005) Inflammationinduced lymphangiogenesis in the cornea arises from CD11bpositive macrophages. J Clin Invest 115:2363-2372

96. Conejo-Garcia JR, Buckanovich RJ, Benencia F et al (2005) Vascular leukocytes contribute to tumor vascularization. Blood 105:679-681

97. Conejo-Garcia JR, Benencia F, Courreges M et al (2004) Tumorinfiltrating dendritic cell precursors recruited by a b-defensin contribute to vasculogenesis under the influence of VEGF-A. Nature Med 10:950-958

98. Li B, Vincent A, Cates JM et al (2009) Low levels of tumor necrosis factor alpha increase tumor growth by inducing an endothelial phenotype of monocytes recruited to the tumor site. Cancer Res 69:338-348

99. Madlambayan GJ, Butler JM, Hosaka K et al (2009) Bone marrow stem and proenitor cell contribution to neovasculogenesis is dependent on model system with SDF-1 as a permissive trigger. Blood 114:4310-4319

100. Lewis CE, De Palma M, Naldini L (2007) Tie2-expressing monocytes and tumor angiogenesis: regulation by hypoxia and angiopoietin-2. Cancer Res 67:8429-8432

101. De Palma M, Venneri MA, Galli R et al (2005) Tie2 identifies a hematopoietic lineage of proangiogenic monocytes required for tumor vessel formation and a mesenchymal population of pericyte progenitors. Cancer Cell 8:211-226

102. Coukos G (2007) Monocytes TIE(2)d up in murky business. Blood 109:5076

103. Venneri MA, De Palma M, Ponzoni M et al (2007) Identification of proangiogenic TIE2-expressing monocytes (TEMs) in human peripheral blood and cancer. Blood 109:5276-5285

104. Pucci F, Venneri MA, Biziato D et al (2009) A distinguishing gene signature shared by tumor-infiltrating Tie2-expressing monocytes, blood "resident" monocytes, and embryonic macrophages suggests common functions and developmental relationships. Blood 114:901-914

105. De Palma M, Venneri MA, Roca C et al (2003) Targeting exogenous genes to tumor angiogenesis by transplantation of genetically modified hematopoietic stem cells. Nat Med 9:789-795

106. Murdoch C, Giannoudis A, Lewis CE (2004) Mechanisms regulating the recruitment of macrophages into hypoxic areas of tumors and other ischemic tissues. Blood 104:2224-2234

107. de Visser KE, Korets LV, Coussens LM (2005) De novo carcinogenesis promoted by chronic inflammation is B lymphocyte dependent. Cancer Cell 7:411-423

108. Pollard JW (2004) Tumour-educated macrophages promote tumour progression and metastasis. Nat Rev Cancer 4:71-78 
109. Schmid MC, Varner JA (2007) Myeloid cell trafficking and tumor angiogenesis. Cancer Letters 250:1-8

110. Mantovani A, Sica A, Allavena P et al (2009) Tumor-associated macrophages and the related myeloid-derived suppressor cells as a paradigm of the diversity of macrophage activation. Hum Immunol 70:325-330

111. Saccani A, Schioppa T, Porta C et al (2006) p50 nuclear factorkappaB overexpression in tumor-associated macrophages inhibits M1 inflammatory responses and antitumor resistance. Cancer Res 66:11432-11440

112. Bray DH, Squire SB, Kawana A et al (1993) Antiretroviral treatment reverses HIV-induced reduction in the expression of surface antigens on alveolar macrophages in AIDS patients. Clin Exp Immunol 91:13-17

113. Chang MK, Raggatt LJ, Alexander KA et al (2008) Osteal tissue macrophages are intercalated throughout human and mouse bone lining tissues and regulate osteoblast function in vitro and in vivo. J Immunol 181:1232-1244

114. Furtado GC, Pina B, Tacke F et al (2006) A novel model of demyelinating encephalomyelitis induced by monocytes and dendritic cells. J Immunol 177:6871-6879
115. Hamann J, Koning N, Pouwels W et al (2007) EMR1, the human homolog of F4/80, is an eosinophil-specific receptor. Eur J Immunol 37:2797-2802

116. Leenen PJ, de Bruijn MF, Voerman JS et al (1994) Markers of mouse macrophage development detected by monoclonal antibodies. J Immunol Methods 174:5-19

117. Ruppert J, Friedrichs D, Xu H et al (1991) IL-4 decreases the expression of the monocyte differentiation marker CD14, paralleled by an increasing accessory potency. Immunobiology 182:449-464

118. Striz I, Pokorna-Sochurkova H, Zheng L et al (2001) Calprotectin expression and mononuclear phagocyte subpopulations in peripheral blood and bronchoalveolar lavage. Sarcoidosis Vasc Diffuse Lung Dis 18:57-63

119. Wilkinson LS, Worrall JG, Sinclair HD et al (1990) Immunohistological reassessment of accessory cell populations in normal and diseased human synovium. Br J Rheumatol 29:259-263

120. Zaba LC, Fuentes-Duculan J, Steinman RM et al (2007) Normal human dermis contains distinct populations of CD11c+BDCA-1+ dendritic cells and CD163+FXIIIA+ macrophages. J Clin Invest 117:2517-2525 